\title{
IMPROVED LUMPING FRICTION MODEL FOR LIQUID PIPE FLOW
}

\author{
Kamil Urbanowicz, ZBigniew ZarzyCKi \\ West Pomeranian University of Technology, Department of Mechanical Engineering and Mechatronics, Szczecin, Poland \\ e-mail: kamil.urbanowicz@zut.edu.pl; zbigniew.zarzycki@zut.edu.pl
}

\begin{abstract}
Normally, during one-dimensional pipe flow, the friction terms are calculated with the use of a numerical method (for example MOC - method of characteristics) at every computational node along the pipe and at every time step. This procedure tends to increase the computational effort greatly. A considerable increase in computational speed can be archived by calculating the frequency-dependent friction at the end of the pipe only. To avoid possible problems (no damping at closed walls, underestimate damping on high impedance components) the frequency-dependent friction term is calculated from the flow waves. The lumping friction model in this work is based on a modificated Schohl convolution integral solution. In addition, the work examined the impact of using of simplified effective weighting function on the obtained results of numerical simulations. The modified method in conjunction with the use of simplified weighting function allow determination of real-time estimate of the basic parameters representing the fluid flow in complex hydraulic systems, water supply, etc.
\end{abstract}

Keywords: transient pipe flow, frequency-dependent friction, effective numerical solution, water hammer, cavitation

\section{Introduction}

In a transient liquid pipe flow velocity and pressure are changing with time. In fluid systems, transient occurs during starting-off or stopping of a pump, closing or opening a valve, or changes in tank levels. Often transient flow conditions persist as oscillating pressure and velocity waves for some time after the initial event that caused it. A water hammer is an example of that condition where the compressibility of the fluid has the dominant effect after a sudden closure of the valve. A transient flow can also result in significant transient pressures that may exceed the design limit of pipes and fittings, especially when the designer calculated it from a very well and most known Joukovsky formula. Detailed understanding of the transient flow is then very important for safe operating of fluid systems.

The hereby study refers to numerical modelling of unsteady flow in pressure conduits. It takes into account the friction loss coefficient as a total of quasi-steady and time-variable coefficient. The time-variable coefficient is calculated with the use of a new effective solution of convolution integral (Urbanowicz and Zarzycki, 2012). The study comprises detailed comparison of numerical results with the use of the conventional method (hydraulic resistance calculated in all computer nodes as a total of two components) and the lumped method (where the resistance coefficient is determined in a simplified way - discussed in detail in the present study), see also Johnston (2006). The results presented in the study show whether the simplified method will be consistent enough to be successfully employed in practice. 


\section{Modelling of the coefficient of friction losses}

During unsteady flow of a liquid in pressure conduits, the value of the friction factor $\lambda$ depends on the averaged flow velocity

$$
\lambda=\lambda_{q}+\frac{32}{|\operatorname{Re}| v} S
$$

where $\lambda_{q}$ is the quasi-steady friction coefficient, Re - Reynolds number, $v$ - average current flow velocity.

On the other hand, the sum $S$ is determined from the below correlation (Urbanowicz and Zarzycki, 2012)

$$
S=\sum_{i=1}^{j} \underbrace{\left[y_{i}(t) A_{i}+\eta B_{i}\left(v_{(t+\Delta t)}-v_{t}\right)+(1-\eta) C_{i}\left(v_{t}-v_{(t-\Delta t)}\right)\right]}_{y_{i}(t+\Delta t)}
$$

where $\eta$ is the corrective coefficient (for details, see Urbanowicz and Zarzycki, 2012), $y_{i}(t)$ - a time-variable parameter which is a value of the above total calculated for the previous time step, $A_{i}, B_{i}$ and $C_{i}$ - constants which are more widely discussed later, $v$-average flow velocity in a proper time stop.

In the laminar flow, the quasi-steady resistance coefficient (the subscript $q l$ ) does not depend on the roughness of the walls and is determined from the below correlation

$$
\lambda_{q l}=\frac{64}{|\operatorname{Re}|}
$$

On the other hand, in the turbulent flow (hence the subscript $q t$ ), the impact of the internal geometrical structure of the walls must not be neglected and it has influence on the resistance coefficient value (Colebrook, 1939)

$$
\frac{1}{\sqrt{\lambda_{q t}}}=-2 \log \left(\frac{2.51}{\operatorname{Re} \sqrt{\lambda_{q t}}}+\frac{\varepsilon / D}{3.7}\right)
$$

where $\varepsilon / D$ is the relative roughness of the pipeline internal walls.

The above empirical Colebrook equation may be solved by numerical methods with high accuracy. There are also analytical approximations of the above formula which determines the friction coefficient in turbulent flows. The best approximation form was given by Goudar and Sonnad (2008) (for details see Appendix A).

The research carried out recently has shown that a sufficient condition for correct (as accurately as required) modelling of hydraulic resistances occurring in course of unsteady states in conduits is constructing the sum $S$ of just two terms. Constants $A_{i}, B_{i}$ and $C_{i}$ occurring in formula (2.2) for this total are calculated from the following correlations

$$
A_{i}=\mathrm{e}^{-n_{i} \Delta \hat{t}} \quad B_{i}=\frac{m_{i}}{\Delta \hat{t} n_{i}}\left(1-A_{i}\right) \quad C_{i}=A_{i} B_{i}
$$

The above correlation shows that the constants $A_{i}, B_{i}$ and $C_{i}$ depend on the dimensionless time step (constant in the method of characteristics) as well as coefficients mi and ni describing the so-called effective weighting function

$$
\Delta \hat{t}=\Delta t \frac{v}{R^{2}} \quad w_{\text {effective }}=\sum_{i=1}^{j} m_{i} \mathrm{e}^{-n_{i} \Delta \hat{t}}
$$


The integral of the aforementioned weighting function multiplied by the derivative of the liquid local velocity is defined as the so-called convolution integral. It is a result of a reverse Laplace transform of the correlation describing the impedance of the hydraulic system presented by Brown (1962). This integral describes the unsteady component of the wall shear stress. It was presented for the first time by Zielke (1968). It takes the following form

$$
\tau_{u}=\frac{2 \mu}{R} \int_{0}^{t} w(t-u) \frac{\partial v}{\partial t}(u) d u
$$

Rquation (2.7) may obviously be used also for the unsteady turbulent flow (Zarzycki, 1997, 2000; Vardy and Brown, 2003, 2004) if the weighting function is known.

The classic solution of the integral (2.7) employed in the method of characteristics is ineffective because the calculation of the momentary value $\tau_{u}$ in the conduit cross section being analysed requires knowledge and use of the entire flow history. Trikha (1975) showed, however, that this solution can be presented in an effective form which allows avoiding the need to store and use the data referring to the average velocity changes occurring in time from the moment of occurrence of the unsteadiness in a specific point of the conduit. In the case of the Trikha method, it is sufficient to know the last two average flow velocities occurring in the current time moment at a specific pipe cross section. Subsequent researchers have perfected the Trikha solution. There are known effective solutions by Kagawa et al. (1983), Schohl (1993) or Urbanowicz and Zarzycki (2012).

The recently carried out benchmark tests of these effective solutions have shown that (Urbanowicz-Zarzycki, 2012):

- the Trikha solution was derived with too many simplifications and it is not suitable for the correct modelling of resistances,

- the solution by Kagawa and others has its classical counterpart in the solution according to Zielke (1968),

- the Schohl solution has its classical counterpart in Zielke-Vardy-Brown solution (Vardy and Brown, 2010) on condition that the weighting function is expressed with a large number of exponential terms (which increases the time of calculation),

- the Urbanowicz-Zarzycki solution is an effective counterpart of the Zielke-Vardy-Brown classical solution but it does not require expressing the effective weighting function with a large number of exponential terms.

Further in the present study, a solution in form of (2.2) presented at Conference of Fluid Mechanics (KKMP2012) held in Gliwice will be used (Urbanowicz-Zarzycki, 2012).

Formula (2.1) shows that when lower velocities are dealt with, bigger is the influence of flow unsteadiness on the momentary value of the resistance coefficient. It can be stated that for large Reynolds numbers $\left(\operatorname{Re}>10^{6}\right)$, the coefficient $\lambda_{u}$ can be entirely negligible since $\lambda_{u} \ll \lambda_{q}$.

\section{Research results}

The correct modelling of hydraulic resistances occurring in hydraulic, water supply pipeline and heating systems in unsteady flow conditions is still not commonly employed in the design stage using specialised software. Most of the common computer programmes continue to be based on an ordinary quasi-steady approach which should not be used in the case of unsteady flows. There is no doubt that the developers of the commercial software dedicated to the modelling of flows are not interested in the correct modelling of resistances because of the mathematical complexity of the known correct solution. Therefore, this study has been dedicated to the comparison of 
the results of numerical research obtained from simplified effective methods of the modelling of the hydraulic resistances. The analysis of literary sources shows that there are two approaches which can possibly be adapted while determining hydraulic resistances with the use of the MOC method of characteristics:

- Standard Effective Method (SM) - the resistances are determined for all analysed cross sections (mostly, there are 16 cross-sections being analysed as the division into such a number ensures that the results obtained are sufficiently accurate) and all time steps with the use of effective solutions of the convolution integral which are based on the effective weighting function, which have been expressed in the previously presented form $(2.6)_{2}$;

- Lumped Friction Method (LFM) - the unsteady hydraulic resistances are determined only in the boundary cross-sections, while only the quasi-steady resistances are determined in the internal cross-sections (Johnston, 2006).

According to the study by Johnston (2006), in the last of the aforementioned approaches, the momentary values of velocities in boundary nodes (Fig. 1) should be corrected in the following manner

$$
v_{R B, c}=\frac{1}{2}\left(v_{R B, r}+\frac{p_{R B, r}}{\rho c}\right) \quad v_{L B, c}=\frac{1}{2}\left(v_{L B, r}+\frac{p_{L B, r}}{\rho c}\right)
$$

where $v_{R B, c}$ and $v_{L B, c}$ are subsequently, velocities corrected at the right and the left boundary, $v_{R B, r}$ and $v_{L B, r}$ - velocities calculated in the classical way on the right and the left boundary, $\rho$ - fluid density, $c$ - pressure wave propagation velocity, $p_{R B, r}$ and $p_{L B, r}-$ pressures at the right and the left boundary of the system, respectively.

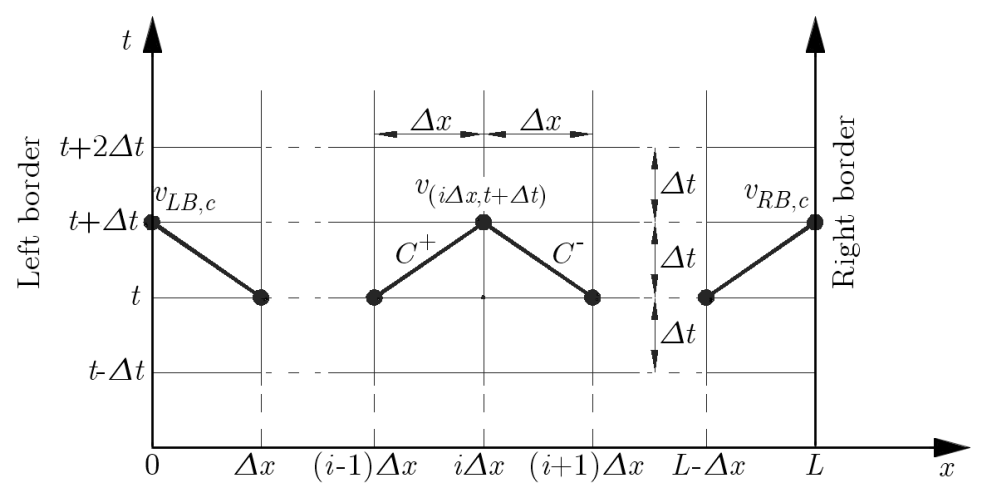

Fig. 1. Method of characteristic grid

The corrected velocity values are used in numerical calculations of the unsteady corrected hydraulic resistance coefficient $\lambda_{u}$, Eqs. (2.1) and (2.2).

The below study examines in detail not only the influence of the method of calculation (the SM standard method or the LFM lumped friction method) but also the correlation between the amount of terms constituting the effective function of weight and the quality of the results obtained. The detailed examination comprises forms of the effective weighting functions composed of the exponential terms 11, 4, 3, 2 - ver. I and 2 - ver. II (for detailed information about the coefficients, see Appendix B). The percentage errors of the adjustment of the above effective weighting functions with respect to Zielke (1968) classical weight are presented in Fig. 2.

The results of the numerical tests carried out with the use of the above methods SM and LFM as well as various forms of the effective weighting function will be compared against the results of experimental tests of pressure change trends in course of hydraulic impact of water hammer. The experimental research was registered by Adamkowski and Lewandowski $(2006,2012)$ at the test facility (Fig. 3) situated at the Institute of Fluid-Flow Machinery of the Polish Academy 


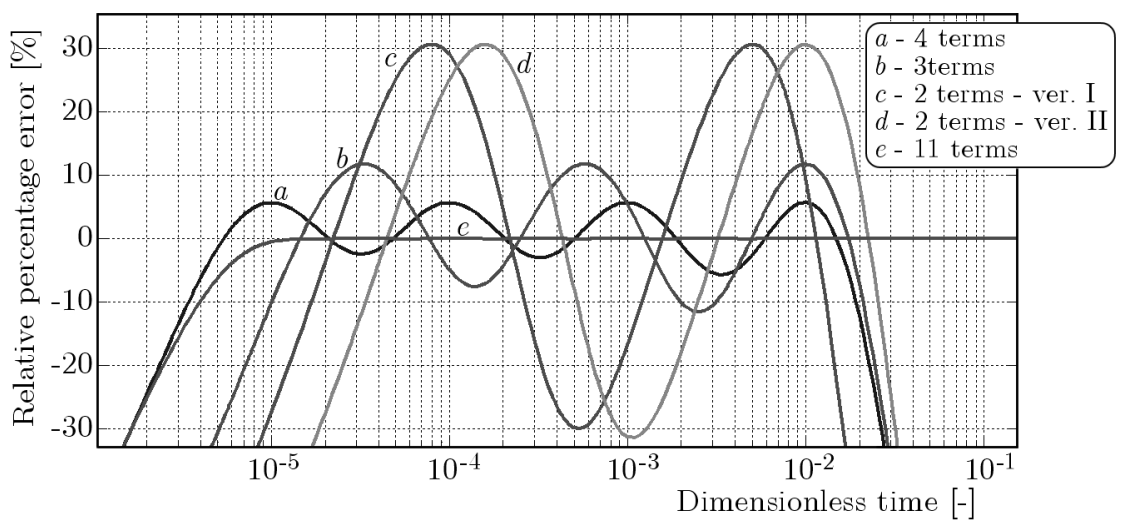

Fig. 2. Effective weighting function errors

of Sciences (PAN) in Gdańsk. This study focused on four experimental courses obtained at the following initial velocities:

- $\operatorname{Re}=1111, v_{0}=0.066 \mathrm{~m} / \mathrm{s}$ (Adamkowski and Lewandowski, 2006)

- $\operatorname{Re}=6899, v_{0}=0.47 \mathrm{~m} / \mathrm{s}$ (Adamkowski and Lewandowski, 2012)

- $\operatorname{Re}=25040, v_{0}=1.7 \mathrm{~m} / \mathrm{s}$ (Adamkowski and Lewandowski, 2012)

- $\operatorname{Re}=40358, v_{0}=2.74 \mathrm{~m} / \mathrm{s}$ (Adamkowski and Lewandowski, 2012)

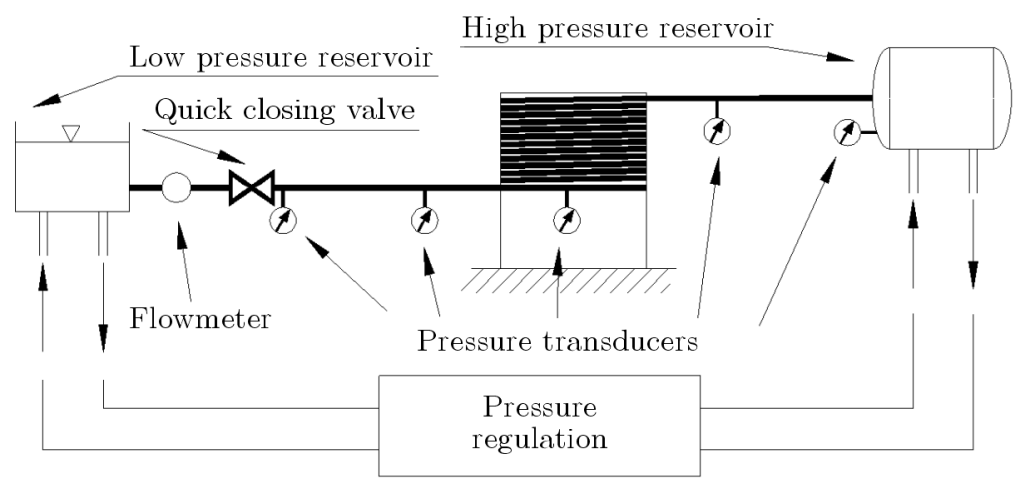

Fig. 3. Layout of the test stand

In the flow in which the phenomenon of liquid column separation occurs, commonly known as vapour cavitation, pressure pulsations can be much greater than those which might be calculated from the commonly used Joukovsky formula

$$
\Delta p=\rho c \Delta v
$$

This is related to the occurrence of sudden accelerations of fluid at the moment when the momentary vapour cavitation areas are closed (Simpson and Wylie, 1991). Therefore, the turbulent courses during which liquid column separation took place play such an important role in this study.

The simulation of unsteady flows with cavitation in the present study employed the use of the cavitation model according to Adamkowski and Lewandowski (2009) in which the artificial dampening taking place in the classical column separation model has been eliminated.

The numerical courses obtained as a result of the simulation have been compared against the experimental courses by means of a simple quantitative method which has been used to 
determine time correctness - the parameter Et (times of occurrence of pressure maximums within subsequent amplitudes of the course of pressure - Fig. 4)

$$
E t=\frac{1}{k} \sum_{i=1}^{k}\left|\frac{t_{i s}-t_{i e}}{t_{i e}}\right| \cdot 100 \%
$$

where $t_{i s}$ are - subsequent times for which a local pressure maximum occurred, $t_{i e}$ - the same times in the experimental course, $k$ - number of pressure amplitudes being analysed.

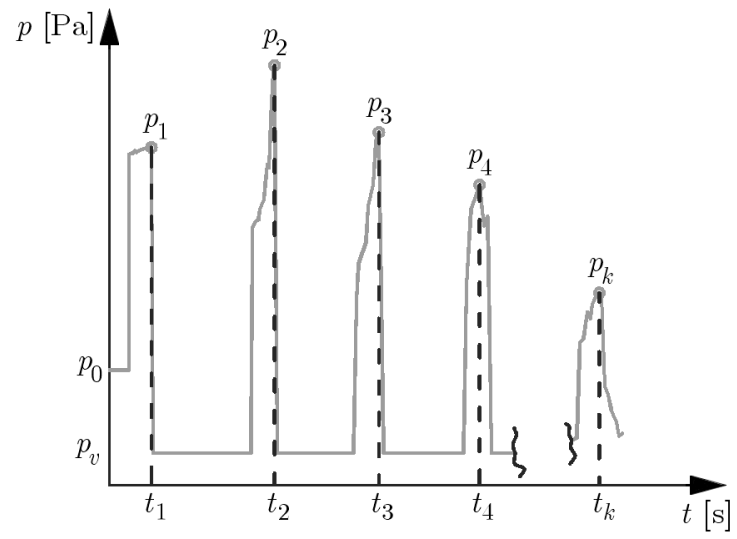

Fig. 4. Analyzed pressures peaks

The value correctness is defined by the parameter $E p$ (the analysis covered values of maximum pressures on subsequent amplitudes - Fig. 4)

$$
E p=\frac{1}{k} \sum_{i=1}^{k}\left|\frac{p_{i s}-p_{i e}}{p_{i e}}\right| \cdot 100 \%
$$

where $p_{i s}$ are maximum values of simulated pressures on subsequent pressure amplitudes, $p_{i e}-$ maximum values of pressures on subsequent amplitudes recorded during the experiment.

The Tables 1-4 include all obtained results of the quantitative analysis. Furthermore, the smallest errors in these tables have been marked bold face to make them attract special attention.

Tabel 1. Errors parameters (for laminar flow $v_{0}=0.0066 \mathrm{~m} / \mathrm{s}$ )

\begin{tabular}{|l|c|c|c|c|}
\hline \multirow{2}{*}{$\begin{array}{c}\text { Type of } \\
\text { weighting } \\
\text { function }\end{array}$} & \multicolumn{2}{c|}{$\begin{array}{c}\text { Standard unsteady } \\
\text { friction - SM }\end{array}$} & \multicolumn{2}{c|}{$\begin{array}{c}\text { Lumped unsteady } \\
\text { friction - LFM }\end{array}$} \\
\cline { 2 - 5 } & $E t$ & $E p$ & $E t$ & $E p$ \\
\hline \hline 2 terms - ver. I & $\mathbf{0 . 6 9 3 3 1 4}$ & $\mathbf{0 . 3 1 8 8 7 6}$ & 0.865383 & $\mathbf{0 . 2 9 2 3 6 4}$ \\
\hline 2 terms - ver. II & 0.693426 & 0.560728 & 0.88126 & 0.430243 \\
\hline 3 terms & 0.703865 & 0.409423 & 0.88045 & 0.465801 \\
\hline 4 terms & 0.702829 & 0.411929 & 0.875647 & 0.514488 \\
\hline 11 terms & 0.702829 & 0.352031 & $\mathbf{0 . 8 6 3 1 4 3}$ & 0.30647 \\
\hline
\end{tabular}

\subsection{Laminar flow results}

The obtained results of the numerical tests for laminar flow show (Table 1) that the simulated changes of pressure by the SM standard method and the LFM method with unsteady resistance 
Tabel 2. Errors parameters (for turbulent flow $v_{0}=0.45 \mathrm{~m} / \mathrm{s}$ )

\begin{tabular}{|l|c|c|c|c|}
\hline \multirow{2}{*}{\begin{tabular}{c} 
Type of $\begin{array}{c}\text { weighting } \\
\text { function }\end{array}$ \\
\cline { 2 - 5 }
\end{tabular}} & \multicolumn{2}{c|}{$\begin{array}{c}\text { Standard unsteady } \\
\text { friction - SM }\end{array}$} & \multicolumn{2}{c|}{$\begin{array}{c}\text { Lumped unsteady } \\
\text { friction }- \text { LFM }\end{array}$} \\
\cline { 2 - 5 } & $E t$ & $E p$ & $E t$ & $E p$ \\
\hline \hline 2 terms - ver. I & $\mathbf{1 . 7 0 0 7 0 4}$ & 17.92573 & $\mathbf{1 . 0 1 0 8 6 2}$ & 15.49663 \\
\hline 2 terms - ver. II & 2.859451 & 17.6086 & 2.333399 & 21.32954 \\
\hline 3 terms & 1.977994 & 16.17788 & 1.177909 & 15.0219 \\
\hline 4 terms & 2.463139 & 17.34088 & 1.784986 & $\mathbf{1 0 . 8 5 2 8 5}$ \\
\hline 11 terms & 2.13715 & $\mathbf{1 5 . 4 1 2 0 2}$ & 1.628331 & 13.00306 \\
\hline
\end{tabular}

Tabel 3. Errors parameters (for turbulent flow $v_{0}=1.7 \mathrm{~m} / \mathrm{s}$ )

\begin{tabular}{|l|c|c|c|c|}
\hline \multirow{2}{*}{$\begin{array}{c}\text { Type of } \\
\text { weighting } \\
\text { function }\end{array}$} & \multicolumn{2}{|c|}{$\begin{array}{c}\text { Standard unsteady } \\
\text { friction }-\mathrm{SM}\end{array}$} & \multicolumn{2}{c|}{$\begin{array}{c}\text { Lumped unsteady } \\
\text { friction }- \text { LFM }\end{array}$} \\
\cline { 2 - 5 } & $E t$ & $E p$ & $E t$ & $E p$ \\
\hline \hline \multicolumn{1}{|c|}{ function } & $E t$ & $E p$ & $E t$ & $E p$ \\
\hline \hline 2 terms - ver. I & 2.979111 & $\mathbf{3 . 3 4 2 3 1 6}$ & 2.269701 & 5.367631 \\
\hline 2 terms - ver. II & 2.696019 & 4.498614 & $\mathbf{1 . 8 5 4 6 3}$ & 6.653467 \\
\hline 3 terms & 2.733686 & 4.344046 & 2.761818 & 5.027982 \\
\hline 4 terms & $\mathbf{2 . 3 2 9 2 5 1}$ & 4.200977 & 2.600806 & $\mathbf{3 . 8 1 2 1 7 3}$ \\
\hline 11 terms & 2.860539 & 3.923151 & 2.419137 & 6.48246 \\
\hline
\end{tabular}

Tabel 4. Errors parameters (for turbulent flow $v_{0}=2.76 \mathrm{~m} / \mathrm{s}$ )

\begin{tabular}{|l|c|c|c|c|}
\hline \multirow{2}{*}{$\begin{array}{c}\text { Type of } \\
\text { weighting } \\
\text { function }\end{array}$} & \multicolumn{2}{|c|}{$\begin{array}{c}\text { Standard unsteady } \\
\text { friction }- \text { SM }\end{array}$} & \multicolumn{2}{c|}{$\begin{array}{c}\text { Lumped unsteady } \\
\text { friction }- \text { LFM }\end{array}$} \\
\cline { 2 - 5 } & $E t$ & $E p$ & $E t$ & $E p$ \\
\hline \hline 2 terms - ver. I & $\mathbf{2 . 1 2 3 9 7 9}$ & $\mathbf{2 . 6 0 6 9 9 9}$ & 2.126099 & 6.09562 \\
\hline 2 terms - ver. II & 2.478371 & 3.962234 & 2.292239 & 3.336577 \\
\hline 3 terms & 2.309905 & 3.883457 & 2.177465 & 4.336589 \\
\hline 4 terms & 2.20718 & 3.934879 & 2.740817 & $\mathbf{1 . 8 6 3 9 7 3}$ \\
\hline 11 terms & 2.20718 & 3.835296 & $\mathbf{1 . 8 7 0 7 1}$ & 4.136423 \\
\hline
\end{tabular}

lumped in boundary nodes (lumped friction model) featured similar accuracy. The average value (computed based on the results obtained for all five effective functions of weigh under analysis) of the parameter $E p$ defining the degree of conformity of the simulated pressure peaks in reference to the experimental results employing the use of the SM and LFM methods was very close to $E p_{\mathrm{SM}}=0.41 \%$ and $E p_{\mathrm{LFM}}=0.40 \%$. The time coincidence of the pressure peaks determined by means of the parameter $E t$, the second parameter calculated in the quantitative method, is slightly worse for the average results obtained by means of the LFM method ( $E t_{\mathrm{SM}}=0.7 \%$ and $\left.E t_{\mathrm{LFM}}=0.87 \%\right)$.

What is interesting is that the best result of the parameter $E p$ is obtained with the use of the LFM method and an effective function of weigh composed of just two terms (2 terms - ver. I). This confirms the fact that, in the case of laminar flows, it is possible to adjust the effective weighting function in such a way that the obtained results will coincide with those obtained with the use of very accurate effective functions of weight (composed of a large number of exponential terms and therefore requiring many more calculations in order to estimate the momentary local resistances). 


\subsection{Turbulent flow results}

a) $v_{0}=0.45 \mathrm{~m} / \mathrm{s}$

The average results calculated from Table 2 shows that the time coincidence of pressure peaks (parametr Et) occurring while using the SM standard method is insignificantly worse $\left(E t_{\mathrm{SM}}=2.23 \%\right)$ than in the case of the LFM method $\left(E t_{\mathrm{LFM}}=1.59 \%\right)$. Even more interesting results have been obtained for the $E p$ parameter which is more important from the practical point of view. As a matter of fact, it can be seen that a similar error has been obtained by using the SM method with a very accurate function of weight $(11$ terms $) E p_{(\mathrm{SM}-11 \text { terms })}=15.4 \%$ as by using the LFM method and a very inaccurate function of weight consisting of just two terms $E p_{(\text {LFM-2 terms ver. I) }}=15.5 \%$.

The course bearing the smallest error is the one simulated by means of the LFM method with the use of a weighting function composed of 4 exponential terms $E p_{\text {(LFM-4terms })}=10.85 \%$ (Fig. 5). It is also worth noticing that the analysis of the first two results obtained by means of the LFM method with the use of weighting functions composed of just two terms shows that the influence of the course of the effective weighting function is crucial; therefore, the span of the result

$$
E p_{(\mathrm{LFM}-2 \text { terms ver. II })}-E p_{(\mathrm{LFM}-2 \text { terms ver. I })}=5.83 \%
$$

obtained between these two examinations is rather large.

At the present stage of the research, it is, however, difficult to determine how to optimally select the shape of the effective weighting function and on what to focus at the stage of determining the coefficients which describe it, so as to ensure that, with a small number of terms, the simplified numerical results are represented with an error similar to that of the accurate results.

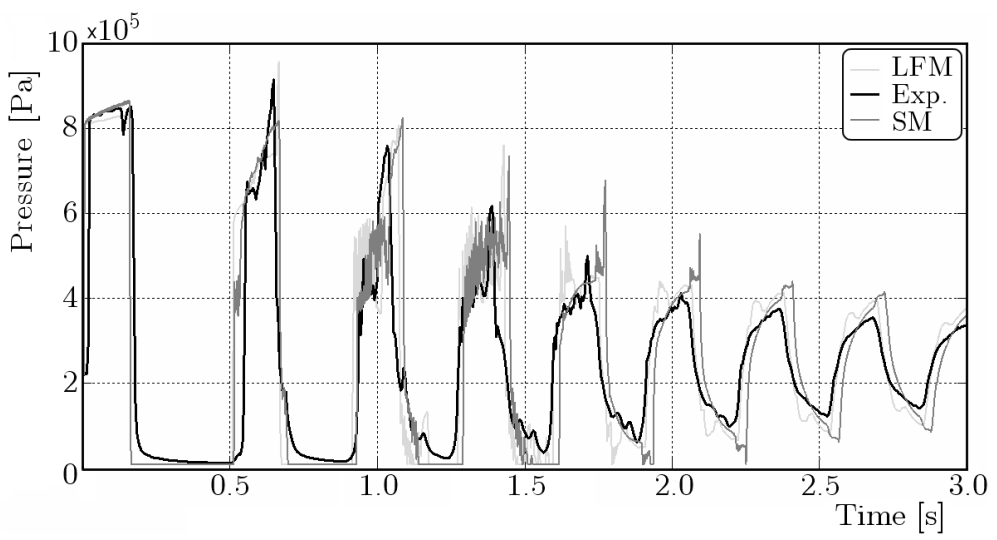

Fig. 5. Pressure variation results at the valve $\left(v_{0}=0.45 \mathrm{~m} / \mathrm{s}, 4\right.$ terms eff. weighting function)

b) $v_{0}=1.7 \mathrm{~m} / \mathrm{s}$

In the analysed case, slightly better average results determining the time coincidence of the occurrence of the pressure course maximum values have been obtained with the use of the simplified LFM method: $E t_{\mathrm{SM}}=2.72 \%$ and $E t_{\mathrm{LFM}}=2.38 \%$. The average values of the parameter $E p$ for the two analysed methods have shown that the SM standard method is better at simulating maximum pressures for higher Reynolds numbers: $E p_{\mathrm{SM}}=4.06 \%$ and $E p_{\mathrm{LFM}}=$ $5.47 \%$. It must not be left unnoticed that also in this case a very good results has been obtained by means of the simplified LFM method and the weighting function composed of four exponential terms: $E p_{(\mathrm{LFM}-4 \text { terms })}=3.81 \%$. The result obtained is lower than the average results, which 
confirms that if the effective weighting function is selected properly, it is possible to obtain a very good course of pressure which is coincident with the experiment by using the simplified LFM method.

c) $v_{0}=2.76 \mathrm{~m} / \mathrm{s}$

The last case of the turbulent flow to be analysed concerns the flow with an initial velocity of $v_{0}=2.76 \mathrm{~m} / \mathrm{s}$. The average values of the parameters $E p$ and $E t$ for the two analysed methods are: $E p_{\mathrm{SM}}=3.64 \%, E p_{\mathrm{LFM}}=3.95 \%, E t_{\mathrm{SM}}=2.26 \%$ and $E t_{\mathrm{LFM}}=2.24 \%$. So, the average results are comparable. This is confirmed by the fact noticed in the course of the analysis of formula (2.1)which becomes more accurate as the average flow velocity increases and the influence of the unsteady resistance coefficient $\lambda_{u}$ decreases. The best results of the parameter $E p$ are again obtained with the use of a weighting function composed of 4 exponential terms $E p_{\text {(LFM-4 terms) }}=$ $1.86 \%$ (Fig. 6), which finally confirms the possibility of commonly using the simplified LFM method while modelling the unsteady hydraulic resistances in pressure conduits.

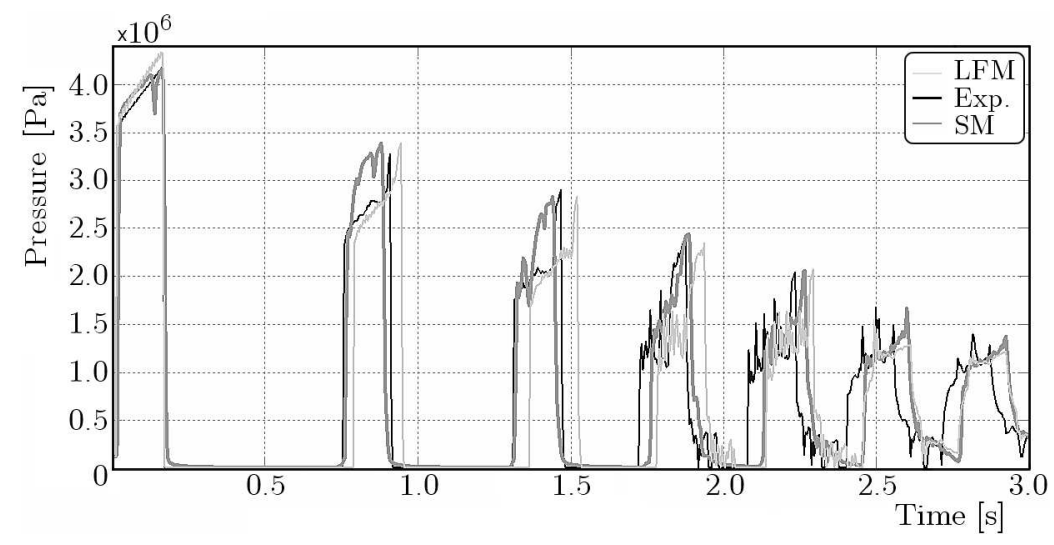

Fig. 6. Pressure variation results at the valve $\left(v_{0}=2.76 \mathrm{~m} / \mathrm{s}, 4\right.$ terms eff. weighting function)

\section{Conclusions}

The presented study compares the research results obtained with the use of the two methods being analysed. In the first of these methods, SM (the standard method), the hydraulic resistance coefficient has been determined in all computational nodes of the methods of characteristics with the use of formula (2.1). In the second method, the so-called lumped method (LFM), the resistance coefficient has been determined with the use of the same formula (2.1), but the sum $S$ found in formula (2.2) is calculated only in the case of boundary nodes while in all internal nodes $S=0$ is implied (that is, as a matter of fact, the hydraulic resistances are determined in these internal nodes in a quasi-steady way).

The results obtained in the study have shown that the simplified LFM method can be used for the modelling of transient pressure courses with a high accuracy. As a matter of fact, using this method, features a degree of accuracy which is close to that obtained when using a method causing much higher computer workload. The results also show that it is possible to achieve very good results in laminar flows by using a very simple effective weighting function composed of just two exponential terms. In the case when the LFM method is used for turbulent flow, the best results would be obtained for an effective weighting function composed of four terms. On the other hand, in the SM standard method, very good results are obtained in the modelling of turbulent flows when simplest weighting function composed of two terms is used.

The results obtained for a flow qualified as turbulent and featuring a small Reynolds number of $\operatorname{Re}=6899$ show too high error level $(E p \approx 15 \%)$. It is difficult to clearly determine the cause 
of such a significant error. It is possible that this is caused by the fact that the Adamkowski and Lewandowski model used for the modelling of sizes of cavitation areas does not take into account the influence of gas cavitation or the nature of unsteady resistances occurring in the course of the transition flow $\left(2320<\operatorname{Re}<10^{4}\right)$ has not been recognised well enough, thus being responsible for the erroneous shape of the weighting function.

At the next stage of the research, it will be necessary to precisely specify the influence of the shape of the effective weighting function. This study shows that a slight offset of the range of usability of the function used (2 terms - ver. I and 2 terms - ver. II) has rather a considerable impact on the obtained results. Determining the influence of the shape of the function will be the last step towards a simple one-dimensional modelling of the hydraulic resistance.

\section{A. Appendix - Goudar-Sonnad turbulent friction factor}

$$
\lambda_{q t}=\left[G_{1}\left(G_{9}+\ln \frac{G_{3}}{G_{5}}\right)\right]^{-2}
$$

where

$$
\begin{aligned}
& G_{1}=\frac{2}{\ln (10)} \quad G_{2}=\frac{\varepsilon / D}{3.7} \quad G_{3}=\frac{\ln (10) \operatorname{Re}}{5.02} \\
& G_{4}=G_{2} G_{3}+\ln G_{3} \quad G_{5}=G_{4}^{G_{4} /\left(G_{4}+1\right)} \quad G_{6}=G_{2} G_{3}+\ln \frac{G_{3}}{G_{5}} \\
& G_{7}=\ln \frac{G_{5}}{G_{6}} \quad G_{8}=G_{7} \frac{G_{6}}{G_{6}+1} \quad G_{9}=G_{8}\left(1+\frac{G_{7} / 2}{\left(G_{6}+1\right)^{2}+\left(G_{7} / 3\right)\left(2 G_{6}-1\right)}\right)
\end{aligned}
$$

\section{B. Appendix - Coefficients of used in simulations effective weighting functions}

2 terms - ver. I: $m_{1}=7.35, m_{2}=61.2, n_{1}=138.2, n_{2}=7984.8$

2 terms - ver. II: $m_{1}=4.9, m_{2}=43.2, n_{1}=79.9, n_{2}=4027$

3 terms: $m_{1}=4.19, m_{2}=17.65, m_{3}=73.6, n_{1}=79.91, n_{2}=1424.8, n_{3}=24788$

4 terms: $m_{1}=3.96, m_{2}=11.97, m_{3}=37.53, m_{4}=118.5, n_{1}=79.91, n_{2}=981.8, n_{3}=10060$, $n_{4}=100896$

11 terms: $m_{1}=1, m_{2}=0.9997, m_{3}=1.0047, m_{4}=1.245, m_{5}=2.328, m_{6}=4.43$, $m_{7}=8.08, m_{8}=14.34, m_{9}=25.15, m_{10}=43.87, m_{11}=76.34, n_{1}=26.3744, n_{2}=70.85$, $n_{3}=135.07, n_{4}=226.2, n_{5}=417.6, n_{6}=946.8, n_{7}=2492.2, n_{8}=7100, n_{9}=20955$, $n_{10}=62745, n_{11}=188918$

The above coefficients of the effective weighting function have been estimated with the use of a computational algorithm described in the study by Urbanowicz (2012).

\section{References}

1. Adamkowski A., Lewandowski M., 2006, Experimental examination of unsteady friction models for transient pipe flow simulation, Journal of Fluids Engineering, 128, 1351-1363

2. Adamkowski A., Lewandowski M., 2009, A new method for numerical prediction of liquid column separation accompanying hydraulic transients in pipelines, Journal of Fluids Engineering, 131, 7, 071302-1-071302-11 
3. Adamkowski A., Lewandowski M., 2012, Investigation of hydraulic transients in a pipeline with column separation, Journal of Hydraulic Engineering, 138, 11, 935-944

4. Brown F.T., 1962, The transient response of fluid lines, Transactions of the ASME, Journal of Basic Engineering, 84, 3, 547-553

5. Colebrook C.F., 1939, Turbulent flow in pipes, with particular reference to the transition region between smooth and rough pipe laws, Journal of the Institution of Civil Engineers, 11, 133-156

6. Goudar C.T., Sonnad J.R., 2008, Comparison of the iterative approximations of the Colebrook-White equation, Hydrocarbon Processing - Fluid Flow and Rotating Equipment Special Report, August, 79-83

7. Johnston D.N., 2006, Efficient methods for numerical modelling of laminar friction in fluid lines, Journal of Dynamic Systems Measurement and Control - Transactions of the ASME, 128, 4, 829-834

8. Kagawa T., Lee I., Kitagawa A., Takenaka T., 1983, High speed and accurate computing method of frequency-dependent friction in laminar pipe flow for characteristics method, Transactions of the Japan Society of Mechanical Engineers, 49, 447, 2638-2644

9. Schohl G.A., 1993, Improved approximate method for simulating frequency - dependent friction in transient laminar flow, Journal of Fluids Engineering, Transactions of the ASME, 115, 3, 420-424

10. Simpson A.R., Wylie E.B., 1991, Large water-hammer pressures for column separation in pipelines, Journal of Hydraulic Engineering, 117, 10, 1310-1316

11. Trikha A.K., 1975, An efficient method for simulating frequency - dependent friction in transient liquid flow, Journal of Fluids Engineering, Transactions of the ASME, March, 97-105

12. Urbanowicz K., 2012, New approximation of unsteady friction weighting functions, Proceedings of the 11th International Conference on Pressure Surges, Lisbon, Portugal, October 24-26, 477-492

13. Urbanowicz K., Zarzycki Z., 2012, Convolution integral in transient pipe flow, Proceedings of XX Fluid Mechanics Conference KKMP2012, Gliwice, Poland, on CD

14. Vardy A.E., Brown J.M.B., 2003, Transient turbulent friction in smooth pipe flows, Journal of Sound and Vibration, 259, 5, 1011-1036

15. VArdy A.E., Brown J.M.B., 2004, Transient turbulent friction in fully rough pipe flows, Journal of Sound and Vibration, 270, 233-257

16. VArdy A.E., Brown J.M.B., 2010, Evaluation of unsteady wall shear stress by Zielke's method, Journal of Hydraulic Engineering, 136, 7, 453-456

17. ZARZYCKi Z., 1997, Hydraulic resistance of unsteady turbulent liquid flow in pipes, Proceedings of 3rd International Conference on Water Pipeline Systems, The Hague, BHR Group, 163-178

18. ZARZYCKI Z., 2000, On weighting function for wall shear stress during unsteady turbulent pipe flow, Proceedings of 8th International Conference on Pressure Surges, BHR Group, The Hague, 529-543

19. Zielke W., 1968, Frequency-dependent friction in transient pipe flow, Journal of ASME, 90, March, 109-115 